EPJ Web of Conferences 52, 09004 (2013)

DOI: $10.1051 /$ epjconf/20135209004

(c) Owned by the authors, published by EDP Sciences, 2013

\title{
Atmospheric leptons
}

\section{the search for a prompt component}

\author{
Thomas K. Gaisser ${ }^{1, a}$ \\ ${ }^{1}$ Bartol Research Institute and Department of Physics and Astronomy \\ University of Delaware, Newark, DE USA
}

\begin{abstract}
The flux of high-energy ( $\geq \mathrm{GeV}$ ) neutrinos consists primarily of those produced by cosmic-ray interactions in the atmosphere. The contribution from extraterrestrial sources is still unknown. Current limits suggest that the observed spectrum is dominated by atmospheric neutrinos up to at least $100 \mathrm{TeV}$. The contribution of charmed hadrons to the flux of atmospheric neutrinos is important in the context of the search for astrophysical neutrinos because the spectrum of such "prompt" neutrinos is harder than that of "conventional" neutrinos from decay of pions and kaons. The prompt component therefore becomes increasingly important as energy increases. This paper reviews the status of the search for prompt muons and neutrinos with emphasis on the complementary aspects of muons, electron neutrinos and muon neutrinos.
\end{abstract}

\section{Introduction}

Before experimental discovery of charmed hadrons at accelerators in the mid-1970s there was intense interest in using atmospheric muons to find evidence for production of heavy, short-lived hadrons. For example, a highlight of the 1973 cosmic-ray conference at Denver was an update of measurements made over several years with the underground muon spectrometer at Park City, Utah [1]. At first, the observed angular dependence of multi-TeV muons had appeared to be more isotropic than could be explained solely by production through the pion and kaon channels, which are strongly enhanced at large zenith angles. With an improved understanding of the overburden and the detector response, however, it was finally concluded that the Park City data were consistent a "conventional" origin from decay of pions and kaons. An isotropic "prompt" component was not manifest for energies below $10 \mathrm{TeV}$.

Production of charmed hadrons has now been measured over a large range of energy at accelerators. The production cross section increases significantly from approximately $\sim 1 \mu \mathrm{b}$ at $\sqrt{s} \approx 10 \mathrm{GeV}$ to several mb at $\sqrt{s} \approx 7 \mathrm{TeV}$ [2]. There is still not full coverage of phase space for charm production, however. In particular, the level of "intrinsic" charm [3] production is still uncertain. The SELEX measurement [4] shows a large asymmetry in the ratio of charm to anti-charm baryons produced by baryon beams on a fixed target, while little or no asymmetry is observed in a pion beam. This observation indicates some level of intrinsic charm in which the valence quarks of the projectile pick up a charmed quark. Charmed

\footnotetext{
a e-mail: gaisser@bartol.udel.edu
}

hadrons produced as fragments of the incident nucleon beam will contribute disproportionately to the spectrum of atmospheric leptons because of the steep cosmic-ray energy spectrum. Thus, even if intrinsic charm contributes less to the total cross section for producing charm than production via QCD processes, it may have a significant effect on the prompt contribution to atmospheric muons and neutrinos.

In addition to the intrinsic interest in identifying the charm contribution to the fluxes of atmospheric muons and neutrinos, there is another, perhaps more important, reason for trying to measure this component. That is because of the relevance of prompt neutrinos in the search for neutrinos of astrophysical origin. Like a flux from unresolved extra-galactic neutrino sources, the prompt contribution is isotropic for $E_{v}<10^{7} \mathrm{GeV}$. It is also harder by one power of energy than the spectrum of conventional atmospheric neutrinos. For these reasons, prompt neutrinos constitute an important background for neutrino astronomy.

The paper begins in $\S 2$ with a discussion of the ingredients needed to calculate fluxes of atmospheric muons and neutrinos, including relevant analytic approximations and the primary cosmic-ray spectrum. Section 3 reviews models for charm production and corresponding predictions for fluxes of muons and neutrinos. In $\S 4$ we calculate the fluxes of conventional atmospheric muons and neutrinos and compare them with the charm contribution. The effect of the knee of the primary spectrum is included. The predictions are illustrated in $\S 5$ with approximate calculations of the event rates for detector with a gigaton target volume like IceCube [5-7]. The concluding Section 6 comments on the current status and prospects for detection of prompt leptons in the near future. 

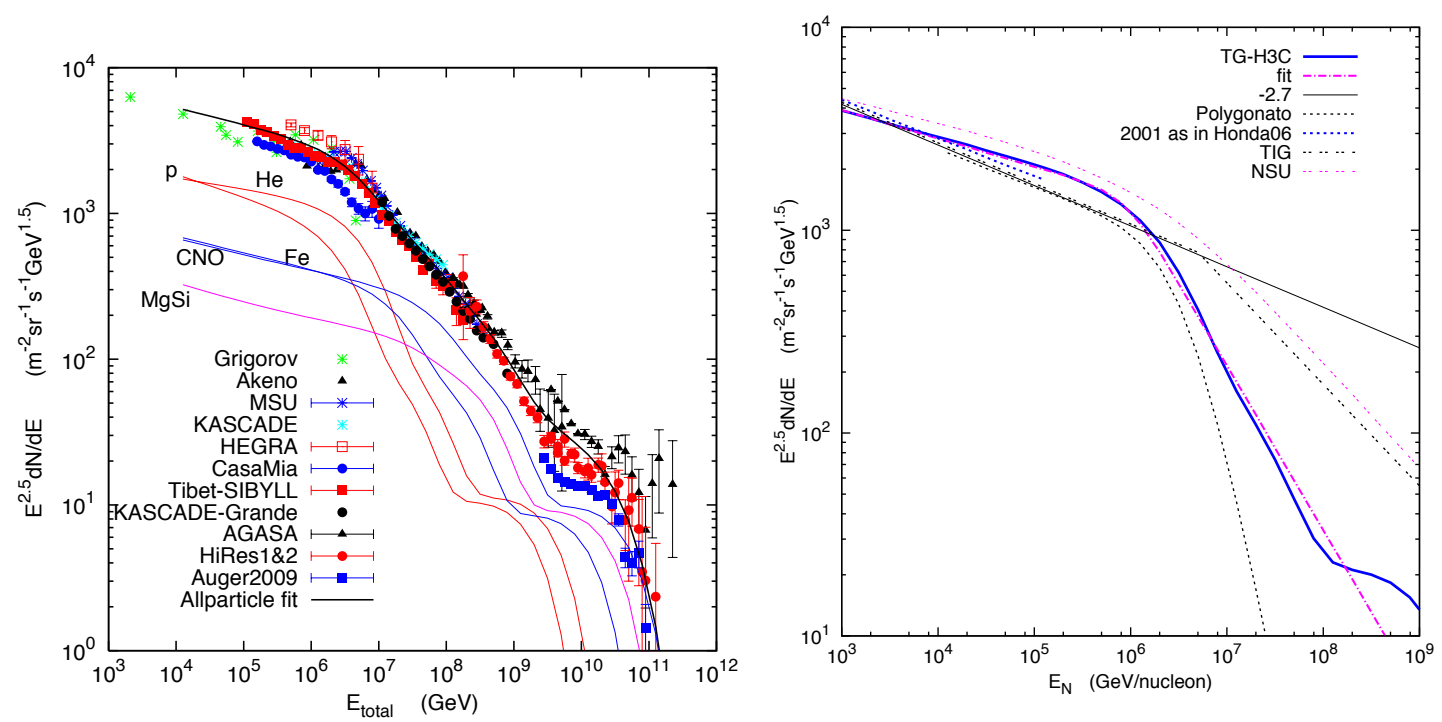

Figure 1. Left: All-particle spectrum from Ref. [8] where references to the data are given. Right: Spectrum of nucleons for several assumptions (see text for explanation).

\section{Atmospheric muons and neutrinos}

The two main ingredients in the calculation of atmospheric neutrinos are the primary spectrum and the hadronic physics of meson production in hadronic interactions. Because production of pions, kaons and charmed hadrons occurs at the nucleon level, what is most relevant is the primary spectrum of nucleons per $\mathrm{GeV} /$ nucleon. Composition comes in through the ratio of protons to total nucleons, which determines the charge ratio of muons and particle/anti-article ratio for neutrinos.

\subsection{Primary spectrum}

For illustration I use a phenomenological model of the primary spectrum with three populations of particles and five nuclear components [8], as shown in Fig. 1 (Left). There are two basic assumptions. First, it is assumed that all energy dependence (whether from acceleration or propagation) depends only on how particles are affected by their magnetic environment. As a consequence, each nuclear component (mass number $Z$ and total momentum per particle $P$ ) depends on magnetic rigidity $(R)$ in the same way, where $R=P c / Z e$. Peters [9] pointed out the consequence of this assumption for the primary composition in the region of the knee of the spectrum, namely, that, when expressed in terms of total energy per particle, protons would steepen first followed by helium and then by nuclei with successively higher charge.

The other assumption, following Hillas [10], is that three populations of particles are sufficient to characterize the entire cosmic-ray spectrum. This is almost certainly an oversimplification. A more realistic picture would likely involve many individual sources injecting particles at various distances and times, as in the model for galactic cosmic rays of Blasi and Amato [11]. Thus the three populations represent three classes of sources:
1. Particles accelerated by supernova remnants in the galaxy,

2. A higher energy galactic component of uncertain origin, and

3. Particles accelerated at extra-galactic sources.

The contribution of nuclei of mass $A_{i}$ to the all-particle spectrum is given by

$$
\phi_{i}(E)=\frac{E \mathrm{~d} N_{i}}{\mathrm{~d} E}=\Sigma_{j=1}^{3} a_{i, j} E^{-\gamma_{i, j}} \times \exp \left[-\frac{E}{Z_{i} R_{c, j}}\right],
$$

where $E$ is the total energy per nucleus.

The spectral indices for each group and the normalizations are given explicitly in Table 1 . The parameters for Population 1 are based on fits to spectra of nuclear groups measured by CREAM $[12,13]$, which we assume can be extrapolated to a rigidity of $4 \mathrm{PV}$ to describe the knee. This is an unverified simplifying assumption that needs to be checked by measurements in the PeV range. In Eq. $1 \phi_{i}$ is $\mathrm{d} N / \mathrm{d} \ln E$ and $\gamma_{i}$ is the integral spectral index. The subscript $i=1,5$ runs over the standard five groups (p, He, $\mathrm{CNO}, \mathrm{Mg}-\mathrm{Si}$ and $\mathrm{Fe}$ ), and the all-particle spectrum is the sum of the five.

The spectrum of nucleons as a function of energy per nucleon corresponding to Eq. 1 is given by

$$
\phi_{N}\left(E_{N}\right)=\frac{E \mathrm{~d} N}{\mathrm{~d} E_{N}}=\Sigma_{i=1}^{5} A_{i} \times \phi_{i}\left(A E_{N}\right) .
$$

Because of the steep cosmic-ray spectrum, protons are relatively more important and heavy nuclei less important in the spectrum of nucleons (as a function of $E_{N}=E_{\text {tot }} / A$ ) than in the all particle spectrum.

The spectrum of nucleons is plotted for several assumptions in Fig. 1 (right). The straight solid line shows a simple $E^{-2.7}$ spectrum of nucleons to guide the eye. The straight dotted line shows the spectrum below $100 \mathrm{TeV}$ 


\begin{tabular}{r|r|ccccc}
\hline$R_{c}$ & $\gamma$ & $\mathrm{p}$ & $\mathrm{He}$ & $\mathrm{CNO}$ & $\mathrm{Mg}-\mathrm{Si}$ & $\mathrm{Fe}$ \\
\hline \hline$\gamma$ for Pop. 1 & -- & 1.66 & 1.58 & 1.63 & 1.67 & 1.63 \\
\hline Population 1: 4 PV & see line 1 & 7860 & 3550 & 2200 & 1430 & 2120 \\
\hline Pop. 2: 30 PV & 1.4 & 20 & 20 & 13.4 & 13.4 & 13.4 \\
Pop. 3 (mixed): 2 EV & 1.4 & 1.7 & 1.7 & 1.14 & 1.14 & 1.14 \\
" (proton only): 60 EV & 1.6 & 200. & 0 & 0 & 0 & 0
\end{tabular}

Table 1. Cutoffs, integral spectral indices and normalizations constants $a_{i, j}$ for Eq. 1 .

recommended in 2001 [14] as a standard for use in calculating fluxes of atmospheric leptons up to $10 \mathrm{TeV}$. At low energy the fit was based on measurements of BESS [15] and AMS [16]. The spectral index used for protons at the time was rather steep (2.74), based on the measurements of BESS and AMS below $200 \mathrm{GeV}$. Recent results of PAMELA [17] show that the spectrum of protons hardens above $200 \mathrm{GeV}$. Two options were given for helium which contributes of order $25 \%$ of the spectrum of nucleons. The high option for helium (with an integral spectral index of $\gamma=1.64$ ) suggested by emulsion chamber measurements in the $10 \mathrm{TeV}$ range at the time $[18,19]$ has since been confirmed by ATIC [20], CREAM [12] and PAMELA [17]. A version of the spectrum of Ref. GHLS is used in the standard calculations of the flux of atmospheric neutrinos by Honda et al. [21] and by the Bartol group [22]. The spectrum of Honda et al. (as described in [23]) uses a harder spectrum for hydrogen (2.71 instead of 2.74) above $100 \mathrm{GeV}$. Their overall spectrum is nearly constant at $\gamma=1.69$ from $200 \mathrm{GeV}$ to $50 \mathrm{TeV}$ with a fraction of helium that increases from $20 \%$ to $25 \%$ in the same region. The spectral index of the spectrum of nucleons in the model of Ref. [8] is nearly constant at $\gamma=1.63$ from $200 \mathrm{GeV}$ to $50 \mathrm{TeV}$ with a corresponding increase in the contribution of helium from $22 \%$ to $30 \%$. The contribution from heavier nuclei is at the level of $10 \%$.

The other lines in the spectrum of nucleons all include the effect of the knee in the cosmic-ray spectrum in one way or another. The heavy solid curve is the nucleon spectrum corresponding to the model in Table 1. The nearby pink dash-dot is an analytic approximation to that model, which is described below in Eq. 13. The strong knee around $1 \mathrm{PeV}$ is the consequence of the increasing fraction of heavy nuclei in the model. In addition to the model of Ref [8], Fig. 1 also shows the polygonato model [24] without any contribution from nuclei heavier than iron. Each nuclear component in the model steepens by $\delta=1.9$ at a rigidity of 4.49 PV. The effect of the knee begins to show up in the nucleon spectrum already somewhat below one $\mathrm{PeV}$. Using the rule of thumb that there is on average a factor of ten between the parent cosmic ray energy and the secondary leptons, taking account of the steepening of the spectrum will be important for muon and neutrino energies of $100 \mathrm{TeV}$ and above, which we is discussed in $\S 4$. The double dotted line that steepens from a differential index of -2.7 to -3.0 at $5 \times 10^{6} \mathrm{GeV}$ is the primary spectrum used in the charm calculation of Ref. TIG.

\begin{tabular}{l|cccc} 
Particle $(\alpha):$ & $\pi^{ \pm}$ & $K^{ \pm}$ & $K_{L}^{0}$ & Charm \\
\hline$\epsilon_{\alpha}(\mathrm{GeV}):$ & 115 & 850 & 205 & $\sim 3 \times 10^{7}$
\end{tabular}

Table 2. Characteristic energies.

\subsection{Hadron production and decay kinematics in the atmosphere}

The phenomenology of atmospheric leptons depends on the production of pions, kaons and heavier hadrons by interactions of cosmic-rays in the atmosphere and on the kinematics for the relevant decay channels. Production and subsequent decay occur through generation by a steep spectrum of primary and secondary cosmic rays in the atmosphere. The competition between reinteraction and decay of unstable hadrons depends on density and altitude. In the framework of a set of analytic approximations for solution of the cascade equations, the essential dependence on energy and zenith angle comes through the critical energy defined as

$$
E_{\text {crit }}=\frac{\epsilon_{\iota}}{\cos \theta^{*}}=\frac{m_{\iota} c^{2} h_{0}}{c \tau_{\iota}},
$$

where the index $\iota$ indicates the hadron $\left(\pi^{ \pm}, K^{ \pm}, K_{L}\right.$ or charmed hadron), $\tau_{\iota}$ is the meson lifetime, $h_{0}$ is the scale height of the atmosphere and $\theta^{*}$ is the zenith angle ( ${ }^{*}$ corrected for curvature of the Earth for $\theta \geq 70^{\circ}$ ). Values of the important characteristic energies are given in Table 2 .

For a power-law spectrum of primary nucleons, the expression for the lepton spectrum factorizes into a product of the primary spectrum and an expression that reflects the properties of production of secondary hadrons by the cosmic-ray spectrum and their subsequent decay to muons and neutrinos.

$$
\begin{aligned}
\phi_{\nu}\left(E_{v}\right)= & \phi_{N}\left(E_{v}\right) \times\left\{\frac{A_{\pi v}}{1+B_{\pi \nu} \cos \theta E_{v} / \epsilon_{\pi}}\right. \\
& +\frac{A_{K v}}{1+B_{K v} \cos \theta E_{v} / \epsilon_{K}} \\
& \left.+\frac{A_{\text {charm } v}}{1+B_{\text {charm } v} \cos \theta E_{v} / \epsilon_{\text {charm }}}\right\},
\end{aligned}
$$

The $A$-factors in Eq. 4 are a product of the spectrum weighted moments for production of mesons by nucleons times the spectrum weighed moments of the meson decay distributions, which include both the decay kinematics and 
the branching ratios. For a power-law spectrum of decaying pions with a differential spectral index $\alpha$ the decay factor is

$$
\frac{1-r_{\pi}^{\alpha}}{\alpha\left(1-r_{\pi}\right)} \text { and } \frac{\left(1-r_{\pi}\right)^{\alpha}}{\alpha\left(1-r_{\pi}\right)}
$$

for muons and neutrinos respectively. In the low energy limit, the spectral index is the same as that of the primary spectrum of nucleons, but at high energy the spectrum of the decaying pions is one power steeper because of the competition between decay and reinteraction. Low and high are with respect to the critical energy $\epsilon_{\pi} / \cos (\theta)$. The ratio $r_{\pi}=m_{\mu}^{2} / m_{\pi}^{2}=0.5731$.

The forms for two-body decay of charged kaons are the same, but the mass ratio factor is much smaller: $r_{K}=$ 0.0458 . The larger critical energy for charged kaons leads to an increase in the contribution of kaons with increasing energy for both muons and neutrinos. The differences between the kinematic factors for two-body decay to neutrinos and muons amplifies the importance of the kaon channel for neutrinos. At high energy, in the $\mathrm{TeV}$ range and above, charged kaons account for about $80 \%$ of muon neutrinos as compared to $25 \%$ of muons.

Each term in Eq. 4 is a form that combines the low energy and high energy solutions to the cascade equation respectively for pions, kaons and charmed hadrons in the atmosphere. The numerator is a product of the spectrum weighted moment for meson production and the spectrum weighted moment of the decay distribution to $v_{\mu}$ [25] with $\alpha=\gamma+1$, where $\gamma$ is the integral spectral index of the spectrum of primary nucleons. The denominator governs the transition between the low and the high energy regimes. The forms for muons are similar. For low energy, meson decay dominates over reinteraction and the resulting lepton spectrum has the same shape as the primary spectrum of nucleons. Charmed hadrons are in the low-energy regime for $E_{\text {lepton }}<10^{7} \mathrm{GeV}$. For high energy $\left(E_{\text {lepton }}>\epsilon_{\alpha} / \cos \theta\right)$, reinteraction of the hadron is more likely and the lepton spectrum becomes one power of energy steeper than the primary spectrum.

In the high energy limit, the spectrum weighted moment for meson decay has to be evaluated on the steeper spectrum, and the attenuation lengths for reinteraction come into play. The $B_{i j}$ quantities in the denominators are the product of the ratios of low-energy to high energy decay distributions combined with the function of attenuation lengths that accounts for cascading of the mesons [26]. Explicitly, for neutrinos

$$
B_{\pi v}=\left(\frac{\gamma+2}{\gamma+1}\right)\left(\frac{1}{1-r_{\pi}}\right)\left(\frac{\Lambda_{\pi}-\Lambda_{N}}{\Lambda_{\pi} \ln \left(\Lambda_{\pi} / \Lambda_{N}\right)}\right)
$$

and for muons

$$
B_{\pi \mu}=\left(\frac{\gamma+2}{\gamma+1}\right)\left(\frac{1-\left(r_{\pi}\right)^{\gamma+1}}{1-\left(r_{\pi}\right)^{\gamma+2}}\right)\left(\frac{\Lambda_{\pi}-\Lambda_{N}}{\Lambda_{\pi} \ln \left(\Lambda_{\pi} / \Lambda_{N}\right)}\right) .
$$

The forms for kaons are the same as functions of $r_{K}$ and $\Lambda_{K}$. The dependence of $\gamma$ on energy in the case of a nonpower law primary spectrum needs to be accounted for.

For a power-law primary spectrum of nucleons and assuming Feynman scaling for hadron production, the cascade equations can be solved analytically as in Eq. 4. The primary spectrum can always be described locally as a power law, and similarly the hadronic interactions can be written in terms of the scaled energy $\left(x=E_{\alpha} / E_{N}\right)$ for a given primary energy per nucleon. In both cases the dependence on energy is sufficiently gradual that the approximate analytical forms can be used for quantitative calculations if the slow variation with energy is accounted for. This approach is taken in the calculation of Thunman, Ingelmann and Gondolo (TIG) [27], which I follow here. They define energy-dependent Z-factors as in the following example for nucleons producing charged pions:

$$
Z_{N \pi^{ \pm}}(E)=\int_{E}^{\infty} \mathrm{d} E^{\prime} \frac{\phi_{N}\left(E^{\prime}\right)}{\phi_{N}(E)} \frac{\lambda_{N}(E)}{\lambda_{N}\left(E^{\prime}\right)} \frac{\mathrm{d} n_{\pi^{ \pm}}\left(E^{\prime}, E\right)}{\mathrm{d} E} .
$$

Here $\lambda_{N}(E)$ is the nucleon interaction length and $\mathrm{d} n_{\pi}^{ \pm}$is the number of charged pions produced in $\mathrm{d} E$ by nucleons of energy $E^{\prime}$, and $\phi_{N}(E)$ is the spectrum of nucleons. The energy-dependent Z-factors are then used in applicable version of Eq. 4 to evaluate the lepton spectrum. This approximation is valid to the extent that the energy dependences are gradual. They showed that the numerical approximation based on the spectrum weighted moments taken from the interaction model used in their Monte Carlo produced similar results to the full Monte Carlo. The advantage is that the analytic approximations can be tuned to match well to a particular model of hadronic interactions. They can then be used to extend a Monte Carlo calculation to arbitrarily high energies without the statistical problems that arise from the fact that mesons usually interact rather than decay at high energy. This method has been used recently in Ref. [28] to complement Monte Carlo calculations of atmospheric leptons in the region of the knee of the cosmic-ray spectrum.

\section{Expectations for prompt leptons}

Limits on the prompt contribution to atmospheric muons are conventionally expressed in terms of the ratio $R_{c}$ of charm production to pion production by rewriting Eq. 4 as

$$
\begin{aligned}
\phi_{\mu}\left(E_{v}\right)= & \phi_{N}\left(E_{\mu}\right) \times\left\{\frac{A_{\pi \mu}}{1+B_{\pi \mu} \cos \theta E_{\mu} / \epsilon_{\pi}}\right. \\
& \left.+\frac{A_{K \mu}}{1+B_{K \mu} \cos \theta E_{\mu} / \epsilon_{K}}+A_{\pi \mu} \times R_{c}\right\}(9)
\end{aligned}
$$

This form applies for lepton energy $<10^{7} \mathrm{GeV}$ where the charm contribution is isotropic. The current upper limit from LVD [29] is $R_{c} \leq 2 \times 10^{-3}$ assuming a differential primary spectrum $\propto E^{-2.77}$. The primary spectrum of nucleons used in this analysis is less steep by about 0.1 , so the LVD limit at $10 \mathrm{TeV}$ (for example) would be reduced by a factor of two for comparison with the models as discussed here. In what follows I will compare the current models for prompt leptons to $R_{c} \simeq 10^{-3}$ considered as an experimental upper limit.

\subsection{Calculations of charm production}

Reference [27] uses a simple Monte Carlo to generate the distribution of hadronic interactions and decays in 


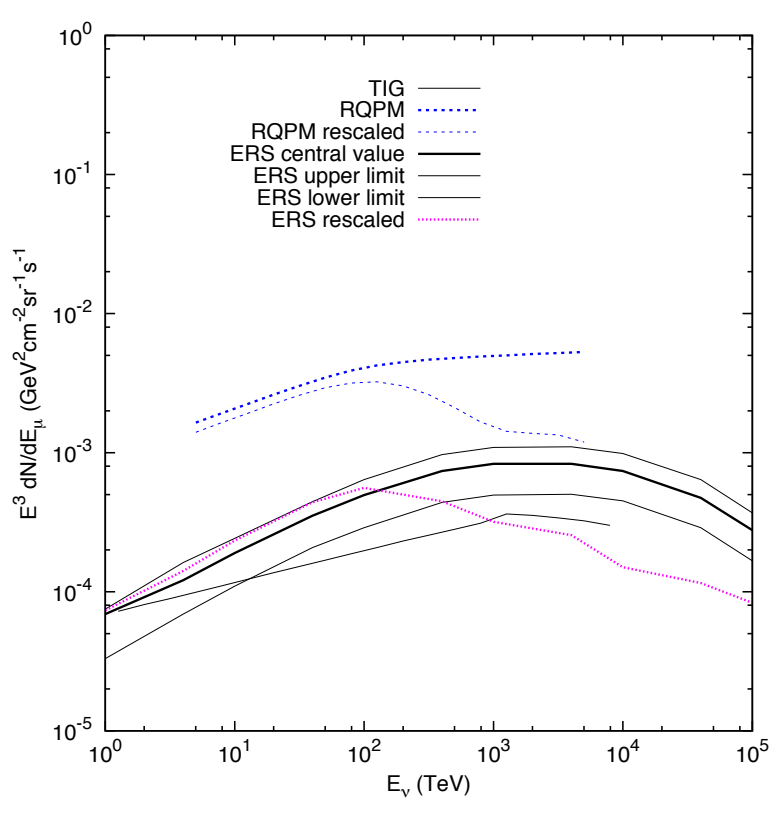

Figure 2. Predictions of three models for the flux of prompt muons. See text for discussion of the rescaled plots.

the atmosphere and Pythia [30] to generate the secondary hadrons at each interaction point. Charm production is calculated within Pythia using first order QCD matrix elements to calculate $c \bar{c}$ production by gluons and by quarks. A renormalization factor $K=2$ is used to represent higher order QCD effects. In addition to their Monte Carlo calculation, They parameterized their results for the charm contribution in a form similar to the third term of Eq. 4 as

$$
\phi_{C}(E)=N_{0} \frac{E^{-(\gamma+1)}}{1+A E}
$$

with $A \approx 3 \times 10^{7} \mathrm{GeV}$ and $\gamma=1.77$ below and $\approx 2.0$ above $10^{6} \mathrm{GeV}$. The value of $R_{c}$ for this model is $\simeq 2 \times 10^{-5}$ at $10 \mathrm{TeV}$.

The reference calculation used for evaluation of the atmospheric neutrino background in IceCube at high energy is that of Enberg, Reno \& Sarcevic (ERS) [31]. It is a QCD calculation that gives results somewhat higher than some previous calculations [27, 32] and lower than an earlier NLO-QCD calculation [33]. The ERS calculation assumes the same primary spectrum of nucleons as in TIG [27], which is shown in the right panel of Fig. 1. The shape of the ERS calculation is similar to that of TIG, and its central value is approximately a factor of two higher. ERS assign an uncertainty range of approximately $\pm 50 \%$ to their calculation. The value of $R_{c}$ for this model is $\simeq 10^{-4}$ at $10 \mathrm{TeV}$.

The "Recombination quark-parton model" (RQPM) [34] embodies the idea of intrinsic charm. The underlying concept is that-also for heavy quarksthere is a process of associated production in which the $c \bar{c}$ pair produced when the projectile proton fragments can recombine with valence quarks (di-quarks) and with sea quarks to produce charmed hadrons, including charmed hyperons. For example, in this model the process $p \rightarrow \Lambda_{c}^{+}+\bar{D}^{0}$ would be expected at a level $\left(m_{s} / m_{c}\right)^{2}$ relative to associated production of strangeness, $p \rightarrow \Lambda K^{+}$. The parameters of the model are adjusted to fit the then available data on charm production in Ref. [35], and a parameterization of the prompt muon flux in the energy range from $5 \mathrm{TeV}$ to $5000 \mathrm{TeV}$ is given. The value of $R_{c}$ for this model is $\simeq 8 \times 10^{-4}$ at $10 \mathrm{TeV}$. The RQPM model is close to the LVD upper limit, but still allowed by it. It is interesting that the recent IceCube limit on the prompt contribution to $v_{\mu}$-induced upward muons [36] is just at the level of the RQPM model.

Figure 2 compares the predictions of these three models for the prompt contribution to the atmospheric muon flux. An important point to note in comparing the curves in this figure is that the spectrum also depends on what is assumed for the primary spectrum. The TIG and ERS use the same assumption as each other, in which the knee is probably too high in energy. The primary spectrum used in Ref. [35] is taken from a model [37] in which the knee is attributed to energy losses in the sources (photodisintegration for nuclei and photo-pion production for nuclei. In this case, the knee is a function of $E / A$ rather than rigidity for the nuclei, and the knee occurs at higher energy per nucleon for protons than for helium because of the relatively high threshold for photo-pion production as compared to photo-disintegration. This spectrum also has a knee at rather high energy and, in addition, appears to be anomalously high even in the few $\mathrm{TeV}$ range, as shown in Fig. 1. The broken lines labeled "rescaled" in the figure are estimates of what the ERS and RQMP models would give for the prompt muon flux if the spectrum of Ref. [8] had been used. This estimate is obtained by multiplying by the ratio at $E_{\mathrm{N}}=10 \times E_{v}$ of the nucleon flux used here [8] to those used for ERS [31] and RQPM [35].

\section{Atmospheric leptons including the knee}

My presentation at ISVHECRI-2012 showed a series of figures in which the contribution of charm was shown separately from the "conventional" atmospheric leptons from decay of pions and kaons. In those figures, a simple power law spectrum was assumed for evaluating the fluxes of the conventional leptons, and the charm contributions were taken directly from the models. Here I take account of the knee in the spectrum, and I make a preliminary effort to present a consistent representation of the prompt component by rescaling their fluxes to the same spectrum of nucleons used for the conventional leptons, as described in the previous paragraph.

Since the primary spectrum is no longer a power law, the spectrum-weighted moments depend on energy. To evaluate energy-dependent Z-factors, Eq. 8 is used. For simplicity here only the energy dependence of the spectrum is considered, and standard values of nucleon interaction and attenuation lengths are used. In addition, a scaling form for meson production is assumed. The goal here is to demonstrate the effect of the knee in the cosmic-ray spectrum on the lepton fluxes. 

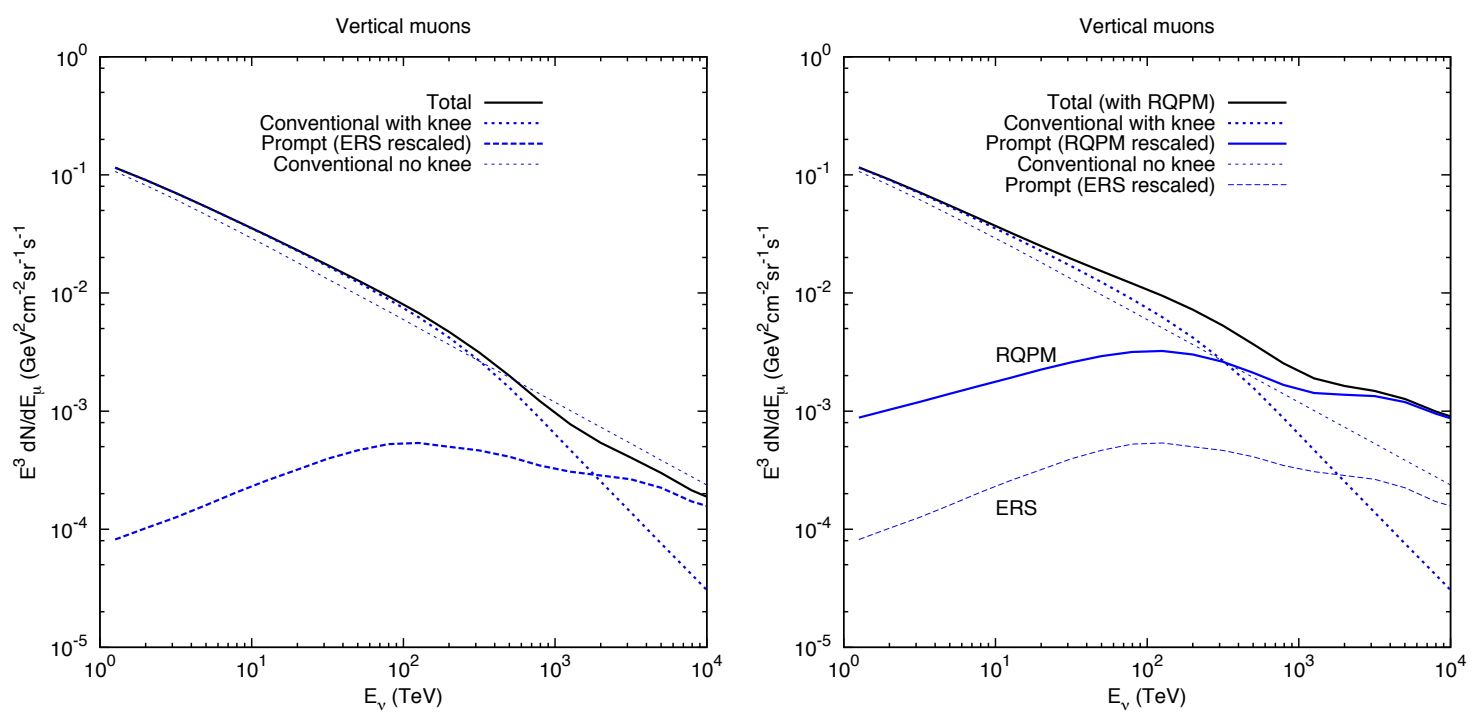

Figure 3. Muon spectra including prompt contribution. Left: prompt component from ERS model (rescaled); Right: prompt component from RQPM model (rescaled).

\subsection{Approximation for pion and kaon production}

Explicit approximations for scale-independent meson production forms are given in Ref. [38]. The approximation for nucleons to produce charged pions is

$F_{N \pi}\left(E_{\pi} / E_{N}\right)=E_{\pi} \frac{\mathrm{d} n_{\pi}\left(E_{\pi} / E_{N}\right)}{\mathrm{d} E_{\pi}} \approx c_{+}(1-x)^{p_{+}}+c_{-}(1-x)^{p_{-}}$

with a similar form for production of kaons. With these scaling forms for particle production, the integral in Eq. 8 can be rewritten as

$$
Z_{N \pi^{ \pm}}=\int_{0}^{1} \frac{\mathrm{d} x}{x^{2}} \frac{\phi_{N}(E / x)}{\phi_{N}(E)} F_{N \pi^{ \pm}}
$$

\subsection{Approximation for nucleon spectrum}

The nucleon spectrum with the knee as parameterized in Table 1 and Eq. 2 can be approximated well (better than $10 \%$ to $30 \mathrm{PeV}$ ) with the standard two-power-law form of Ref. [39] to describe the knee, a form which is also used in Ref. [24]. Specifically,

$$
E \frac{\mathrm{d} N}{\mathrm{~d} E}=\text { const } \times E^{-\gamma}\left(1+\left(E / E^{*}\right)^{\epsilon}\right)^{-\delta / \epsilon},
$$

with $\gamma=1.64, \delta=0.67, E^{*}=9 . E+5 \mathrm{GeV}$ and $\epsilon=3.0$. This approximation locates the knee in the nucleon spectrum just below a PeV, and the slope steepens from an integral spectral index of 1.64 below the knee to 2.31 after the knee. The normalization constant is $10290 . \mathrm{m}^{-2} \mathrm{sr}^{-1} \mathrm{~s}^{-1}$. The steepening in the nucleon spectrum is a consequence of the steepening of the all-particle spectrum amplified by the increasing fraction of nuclei in the all-particle spectrum. Equations 11, 12 and 13 are combined and integrated numerically to obtain energy-dependent Z-factors.

\subsection{Atmospheric leptons including the knee}

The fluxes of $\mu^{ \pm}, v_{\mu}+\bar{v}_{\mu}$ from decay of pions and kaons are obtained using the energy-dependent Z-factors to evaluate Eq. 4. The energy-dependence of the spectral index in the meson decay factors that appear in Eq. 4 are also accounted for by using the local (energy-dependent) integral spectral index of the nucleon spectrum, which steepens gradually from $\gamma=1.64$ to 2.31 through the knee region.

Calculation of the flux of electron neutrinos requires tracing the contributions of Ke3 decays of both charged and neutral kaons. This has been done by taking account of the neutron/proton ratio of the primary nucleons and tracking separately the production of $K \bar{K}$ pairs and production of kaons in association with $\Lambda$ and $\Sigma$ hyperons. An approximate value of $n / p=0.54$ has been used [8]. As noted in the previous section, associated production of kaons by dissociation of an incident nucleon into a kaon and a hyperon is a prototype for intrinsic charm.

Results for the lepton fluxes are shown in Figs. 3 and 4. The vertical muon fluxes are shown in Fig. 3, comparing the ERS model for prompt muons (left) to the RQPM model (right). Below the knee the RQPM prompt flux is rescaled down by about $15 \%$, while the ERS prompt muon flux is rescaled up by about $20 \%$. The rescaled ERS prompt muons are repeated on the right panel for comparison. In the region between $100 \mathrm{TeV}$ and $1 \mathrm{PeV}$ the rescaled RQPM prompt flux is a factor of 5 to 6 higher than the rescaled ERS flux. The RQPM prompt component crosses the conventional flux around $300 \mathrm{TeV}$, as compared to a crossover at $2 \mathrm{PeV}$ for ERS (left panel).

Figure 4 compares the situation for $v_{\mu}$ (left panel) with that for $v_{e}$ (right panel). The ERS model is shown for the prompt component. The RQPM flux is also repeated on both plots for reference. The conventional atmospheric $v_{e}$ 

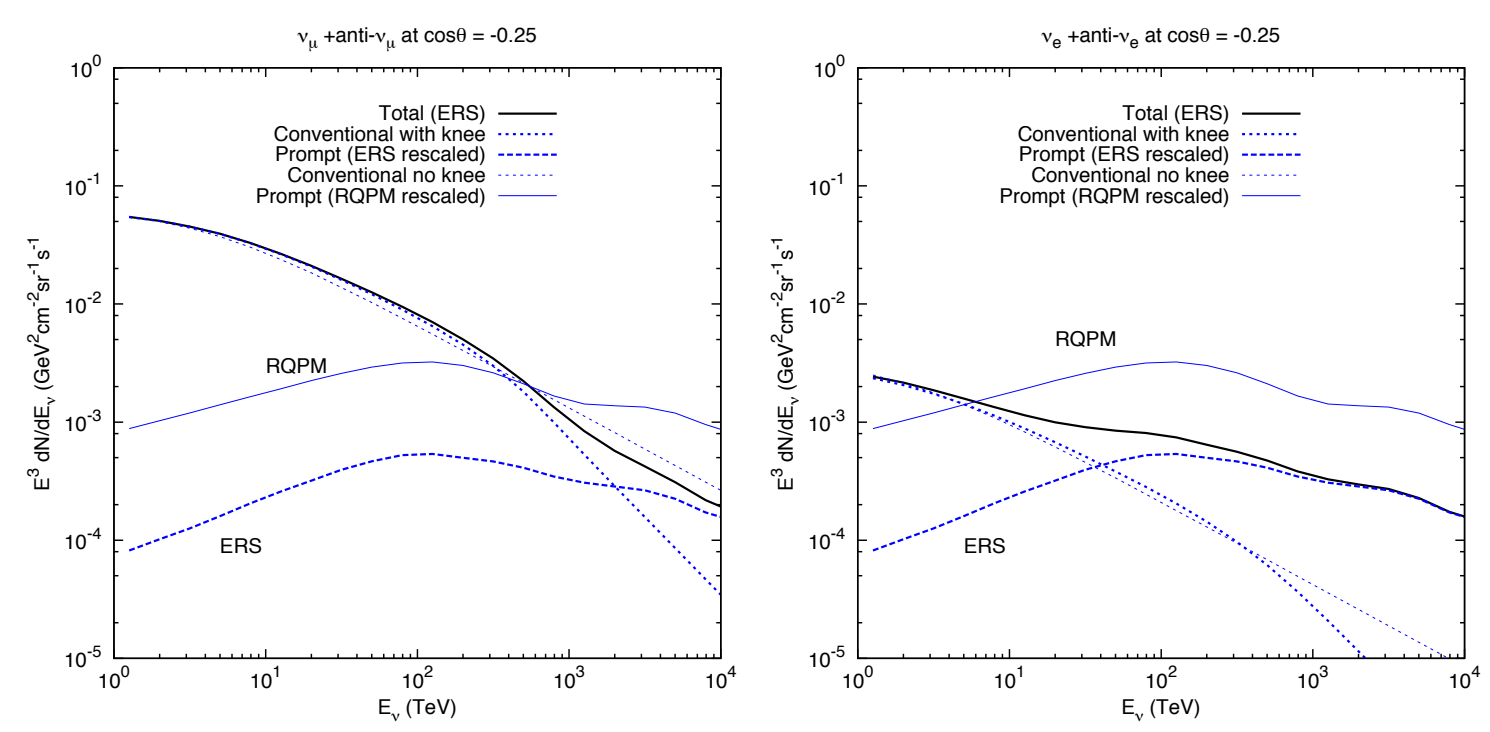

Figure 4. Neutrino spectra including the prompt contribution. Left: $v_{\mu}+\bar{v}_{\mu}$; Right: $v_{e}+\bar{v}_{e}$.

flux is approximately a factor 20 lower than the conventional flux of $v_{\mu}$, so the electron neutrino component is dominated by the prompt component at quite low energy.

\section{Expected rates}

The fluxes described above can be used to estimate the rate of events in a kilometer scale detector. For atmospheric muons the rate per year is simply the flux multiplied by $3 x$ $10^{7}$ seconds/yr and by $10^{10} \mathrm{~cm}^{2} / \mathrm{km}^{2}$. The corresponding integral rate of events $I_{\mu}\left(>E_{m} u\right)$ is shown in Fig. 5 .

The rate of neutrino-induced muons can be obtained in a similar way, with one additional step. It is necessary to calculate the effective area to convert the rate of neutrinos with trajectories passing through the detector to a rate of neutrino-induced muons. Effective area is defined in such a way that $\phi\left(E_{v}, \theta\right) \times A_{\text {eff }}\left(E_{v}, \theta\right)$ is the rate of neutrino-induced muons per second per sr at zenith angle $\theta$. Explicitly

$A_{\mathrm{eff}}\left(E_{v}, \theta\right)=\epsilon\left(E_{\mathrm{th}}, \theta\right) A(\theta) P_{v}\left(E_{v}, E_{\mathrm{th}}\right) \exp \left\{-\sigma_{v}\left(E_{v}\right) N_{A} X(\theta)\right\}$,

where $P\left(E_{v}\right)$ is the probability that a neutrino converts and produces a muon that reaches the detector with enough energy to be reconstructed. Absorption of neutrinos in the Earth becomes significant in the 10 to $1000 \mathrm{TeV}$ range, first for vertically upward trajectories and for neutrinos with zenith angles $\sim 120^{\circ}$ around a PeV. An accurate calculation of $A_{\text {eff }}$ requires a detector simulation. Here I use an estimate for an ideal $\mathrm{km}^{2}$ detector from Ref. [40] and estimate the rate of neutrino-induced muons in the zenith angle range from horizontal to $-120^{\circ}$. The result is shown for the ERS assumption in the left panel of Fig.!6.

Electron neutrinos must interact in the detector to be identified as cascades in the detector. Such cascades are virtually indistinguishable from neutral current interactions of muon or electron neutrinos of energy $E_{v} \sim E_{v_{e}} / y$, where $y$ is the inelasticity of the neutral current neutrino interaction. The neutral current interactions of atmospheric $v_{\mu}$ make a comparable contribution to cascades for the conventional atmospheric neutrinos because the flux of $v_{\mu}$ is significantly higher than that of $v_{e}$. For the charm component, however, the neutral current contribution is relatively unimportant because of the equality of the fluxes of prompt $v_{e}$ and $v_{\mu}$. The integral rate of $v_{e}$ interactions from all directions is

$$
R\left(>E_{v}\right)=4 \pi N \times T \times \int_{E_{v}} \sigma_{\mathrm{cc}} \phi\left(E_{v}\right) \mathrm{d} E_{\nu},
$$

where $\phi\left(E_{v}\right)$ is the spectrum of $v_{e}+\bar{v}_{e}$ averaged over all directions and $\sigma_{\mathrm{cc}}$ is the charged current cross section, taken here from Ref. [41]. $\mathrm{N}$ is the number of nucleons per cubic kilometer of ice and $\mathrm{T}=1$ year. The resulting estimate of the rate of atmospheric $v_{e}$ interactions is shown in the right panel of Fig. 6. The plot includes the effect of neutrino shadowing by the Earth in the upward hemisphere [42]. This amounts to a suppression for the whole sky of $9 \%$ at $100 \mathrm{TeV}$ and $23 \%$ at a PeV. The plot shows about 5 electron neutrino interactions above $100 \mathrm{TeV}$ per $\mathrm{km}^{3}$ of ice per year assuming ideal (full) efficiency. The spectrum is steeply falling so that less than one such event in ten years is expected above a PeV.

\section{Summary comments}

In view of the recent observation by IceCube of two cascade-like events with observed energy just above a PeV [43], together with the observation of several high=energy cascades reported at this meeting [44], it is important to achieve a good understanding of the background from atmospheric neutrinos in the energy region around $100 \mathrm{TeV}$ and above. The work outlined in this paper is just a start. 

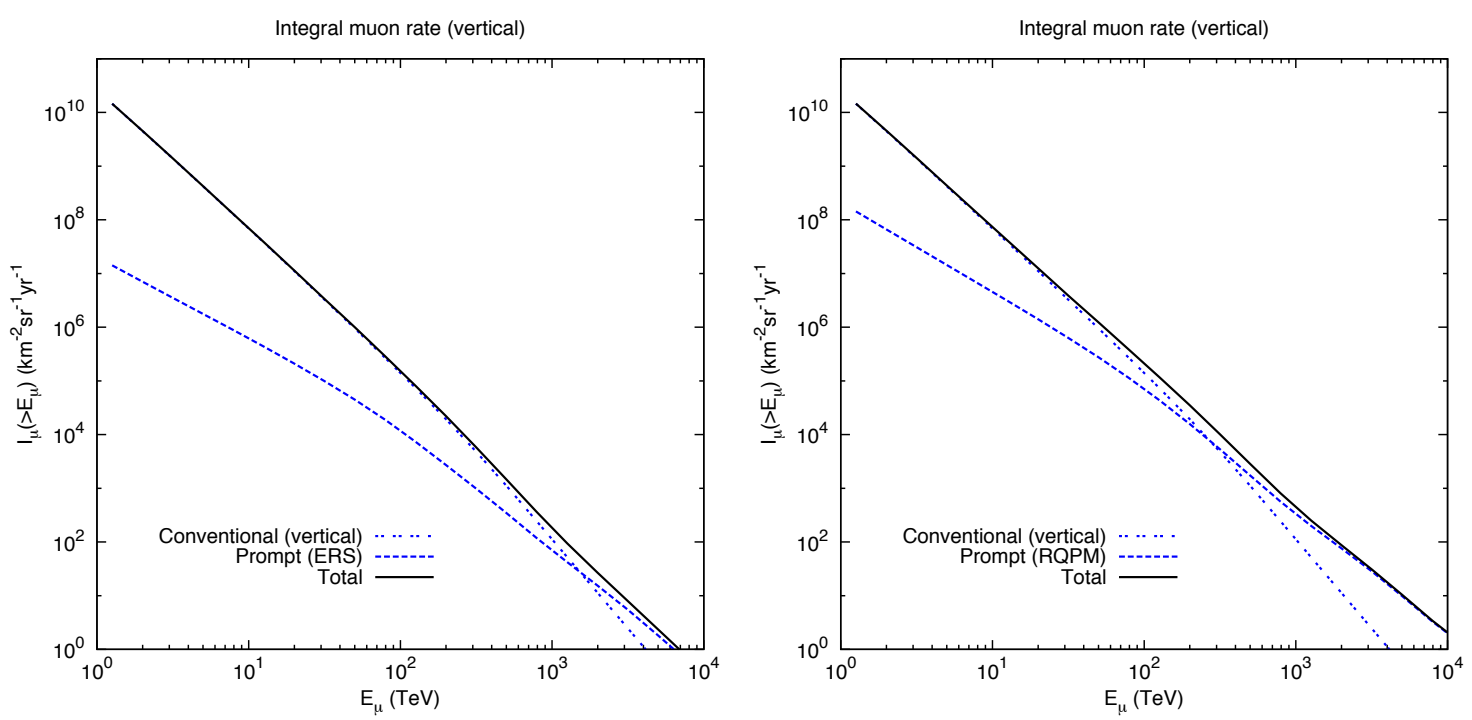

Figure 5. Integral muon rate. Left: with rescaled ERS model for prompt muons; Right: with rescaled RQPM model for charm.
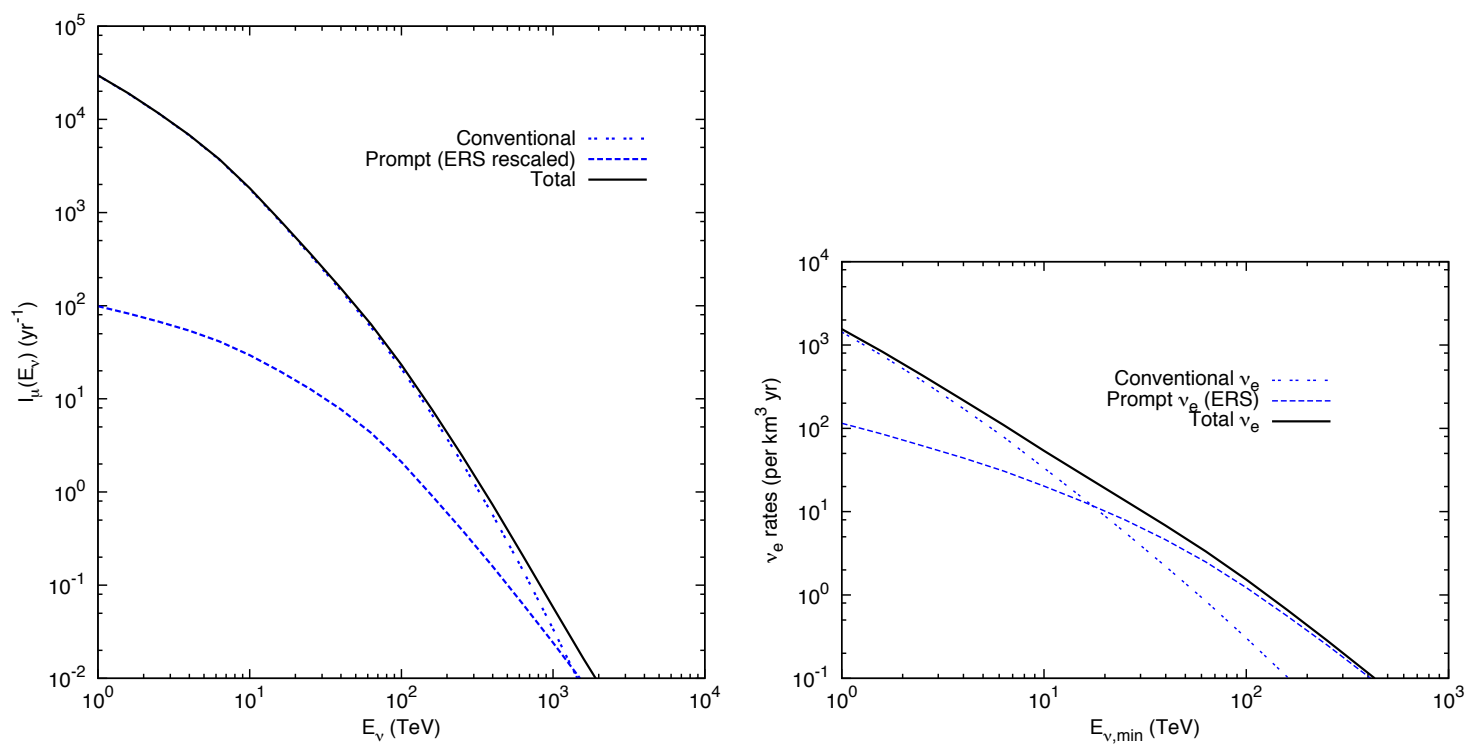

Figure 6. Estimate of the rate of atmospheric neutrino interactions per year in a km3 detector. Left: neutrino-induced muons per $\mathrm{km}^{2}$ and with zenith angles from $90^{\circ}$ to $120^{\circ}$; Right: electron neutrinos with vertices inside $1 \mathrm{~km}^{3}$. ERS is rescaled in both plots, as discussed in the text.

There are large uncertainties in the primary spectrum in the $\mathrm{TeV}$ range and above that need to be assessed. The model used here is just one possibility. A model for charm production that includes recent data from LHC $[45,46]$ in its fits needs to be developed. As an example, the framework for including charm within SIBYLL [48] exists [49], but the parameters need to be tuned to data over a wide range of energy and phase space. An updated model could then be used for a full Monte Carlo simulation including charm. The analytic approximations will remain an important tool to supplement the Monte Carlo, for example to track the consequences of uncertainties in the input spectrum and hadronic interactions for the expected fluxes [47], as well as to parameterize and extrapolate the limited statistics of the full Monte Carlo [28].

\section{Acknowledgments}

I am grateful to colleagues in the IceCube collaboration for discussions in various contexts that stimulated this work. My research related to IceCube is supported in part by NSF-PHY-1205809. My research related to hadronic interaction models is supported in part by the U.S. Department of Energy, DE-SC0007893. 


\section{References}

[1] H.E. Bergeson, et al., Proc. 13th Int. Cosmic Ray Conf. (Denver), 3, 1722 (1973).

[2] Y. Pachmayer (ALICE Collaboration) arXiv:1110.6462.

[3] S.J. Brodsky et al., Phys. Lett. B 93, 451 (1980).

[4] SELEX Collaboration, (F.G. Garcia et al.,) Phys. Lett. B 528, 49 (2002).

[5] F. Halzen in Proc. IAU Symposium 288 "Astrophysics from Antarctica" (Cambridge University Press, ed. M.G. Burton, X. Cui \& N.F.H. Tothill, Cambridge University Press, 2013), pp. 84-97.

[6] A. Karle, Ibid., pp. 98-104.

[7] T.K. Gaisser (for the IceCube Collaboration), Ibid., pp. 105-114.

[8] T.K. Gaisser, Astropart. Phys. 35 (2012) 801-806.

[9] B. Peters, Il Nuovo Cimento XXII (1961) 800-819.

[10] A.M. Hillas, arXiv:astro-ph/0607109.

[11] P. Blasi \& E. Amato, arXiv:1 105.4521.

[12] H.S. Ahn et al., Ap.J. 714 (2010) L89-L93.

[13] H.S. Ahn, et al., Ap.J. 707 (2009) 593-603.

[14] T.K. Gaisser, M. Honda, P. Lipari \& T. Stanev, Proc. ICRC2001 (Hamburg, 2001), vol. 5, p. 1643.

[15] T. Sanuki et al., Ap. J. 545 (2000) 1135.

[16] J. Alcaraz et al., Phys. Lett. B 490 (2000) 27.

[17] O. Adriani et al., Science 332 (2011) 6025.

[18] M. Ichimura et al., Phys. Rev. D 48 (1993) 19491976.

[19] K. Asakimori et al., Ap. J. 502 (1998) 278-283.

[20] D. Panov et al., Bull. Russian Acad. Sci. Phys. 73 (2009) 564-567.

[21] M. Honda et al., Phys. Rev. D 75 (2007) 043006.

[22] G.D. Barr, S. Robbins, T.K. Gaisser \& T. Stanev, Phys. Rev. D74 (2006) 094009.

[23] M. Honda, T. Kajita, K. Kasahara \& S. Midorikawa, Phys. Rev. D70 (2004) 043008.

[24] J.R. Hörandel, Astropart. Phys. 19 (2003) 193.
[25] P. Lipari, Astropart. Phys. 1 (1993) 195-

[26] T.K. Gaisser Cosmic Rays and Particle Physics (Cambridge University Press, 1990).

[27] M. Thunman, G. Ingelman \& P. Gondolo, Astropart. Phys. 5 (1996) 309-332.

[28] A. Fedynitch, J. Becker Tjus, \& P. Desiati, Phys. Rev. D86, 114014 (2012).

[29] M. Aglietta et al., Phys. Rev. D 60 (1999) 112001.

[30] Pythia reference

[31] R. Enberg, M.H. Reno \& I. Sarcevic, Phys. Rev. D 78, 043005 (2008).

[32] A.D. Martin, M.G. Ryskin \& A.M. Stasto, Acta Phys. Polon. B 34, 3273 (2003).

[33] L. Pasquali, M.H. Reno \& I. Sarcevic, Phys. Rev. D 59, 034020 (1999).

[34] E.V. Bugaev et al., Nuovo Cimento C 1241 (1989).

[35] E.V. Bugaev et al., Phys. Rev. D 58, 054001 (1998).

[36] Anne Schukraft (for the IceCube Collaboration), Proc. NOW 2012 (arXiv:1302.0127).

[37] S.N. Nikolsky, I.I. Stamenov \& S.Z. Ushev, Sov. Phys. JETP 60, 10 (1984).

[38] T.K. Gaisser, Astropart. Phys. 16 (2002) 285-294.

[39] S. V. Ter-Antonyan and L. S. Haroyan, arXiv:hepex/0003006v3 (2000).

[40] T.K. Gaisser, Earth Planets Space 62, 195 (2010).

[41] A. Cooper-Sarkar, Philipp Mertsch \& S. Sarkar, JHEP 08 (2011) 042 (arXiv:1106.3723v2).

[42] R. Gandhi, et al., Astropart. Phys. 5, 81 (1996).

[43] Aya Ishihara (for the IceCube Collaboration), Proc. Neutrino 2012.

[44] Eike Middell (for the IceCube Collaboration), Presentation at this meeting (ISVHECRI2012).

[45] The ALICE Collaboration, arXiv:1111.1553v2

[46] LHCb Collaboration, arXiv:1302.2864.

[47] V. Agrawal et al., Phys. Rev. D53, 1314 (1996).

[48] E.-J. Ahn et al., Phys. Rev. D 80, 094003 (2009).

[49] E.-J. Ahn et al., arXiv:1102.5705. 